\title{
Manifestations ouvrières et théories de la violence
} : 1919-1934

\section{Danielle Tartakowsky}

\section{(2) OpenEdition \\ Journals}

Édition électronique

URL : http://journals.openedition.org/conflits/215

DOI : $10.4000 /$ conflits.215

ISSN : $1777-5345$

Éditeur :

CCLS - Centre d'études sur les conflits lilberté et sécurité, L'Harmattan

Édition imprimée

Date de publication : 15 mai 1993

ISSN : 1157-996X

\section{Référence électronique}

Danielle Tartakowsky, « Manifestations ouvrières et théories de la violence : 1919-1934 », Cultures \&

Conflits [En ligne], 09-10 | printemps-été 1993, mis en ligne le 04 mars 2005, consulté le 30 mars 2021. URL : http://journals.openedition.org/conflits/215 ; DOI : https://doi.org/10.4000/conflits.215

Ce document a été généré automatiquement le 30 mars 2021.

Creative Commons License 


\title{
Manifestations ouvrières et théories de la violence : 1919-1934
}

\author{
Danielle Tartakowsky
}

1 Certaines composantes du mouvement ouvrier français se réclament explicitement de la violence à deux reprises de 1919 au début des années trente. Il s'agit des syndicalistes révolutionnaires au sortir de la guerre puis du Parti communiste entre 1929 et 1931. Nous voudrions analyser les finalités que ces organisations assignent à la violence, en comparer les termes et nous interroger sur les relations qu'entretiennent leurs théories avec la violence effective de certaines manifestations ouvrières qui se déploient ces années durant. Les limites imparties à ce texte nous amèneront à raisonner principalement à partir de ces cas paroxysmiques que sont les manifestations ayant entraîné mort d'hommes ou ayant vu surgir des barricades. "La force a pour objet d'imposer l'organisation d'un certain ordre social dans lequel une minorité gouverne [quand] la violence tend à la destruction de cet ordre" écrit, avant guerre, Sorel. La force cherche à réaliser une obéissance automatique; la violence veut briser cette autorité"1. Le propos résume à la perfection les conceptions de la rue qui s'opposent au sortir de la première guerre mondiale. Les socialistes et syndicalistes majoritaires tiennent les manifestations qu'ils organisent pour l'instrument privilégié d'une démonstration de force destinée à peser sur et dans le système existant; les minoritaires, qui seuls nous retiendrons ici, les conçoivent au contraire comme l'occasion parmi d'autres d'une violence dont la "rue" se veut le simple synonyme.

\section{LES SYNDICALISTES REVOLUTIONNAIRES}

3 La violence ainsi comprise n'est qu'une des formes de l'action directe dont ils se réclamaient avant guerre. Certains veulent y voir l'occasion d'une fraternisation avec l'armée et d'autres le moyen d'enclencher le processus pouvant conduire au "grand soir"2. Les objectifs qu'ils lui assignent répondent de ce qu'ils refusent les interdictions dont leurs rassemblements font l'objet en assumant la probable violence qui découle de 
leur maintien. "On forcera les barrages" déclarent certains dirigeants de la région parisienne à la veille du premier mai 19193. Et d'autres: "Les bourgeois ont peur, le gouvernement tremble; quant aux flics, ils savent depuis la manifestation Jaurès comment les cheminots se défendent si on veut les empêcher de manifester pour la justice et pour le droit. Tous avec des bannières syndicales! Si on veut nous barrer la route, nous cognerons !" ${ }^{4}$. Les métallurgistes de l'Isère promettent pareillement, en décembre, d'être calmes "si la police ne les tracasse pas" mais se refusent à répondre à d'éventuels incidents dans le cas contraire : "c'est moi qui porterait le drapeau, déclare leur secrétaire. Si la police enlève l'étoffe, ils n'enlèveront pas la hampe"5. Un jeune délégué qui assiste pour la première fois à la $\mathrm{CE}$ de l'union des syndicats de la Seine affirme encore, nonobstant les récentes leçons du premier mai, qu'il sortira le 21 juillet ${ }^{6}$ dans la rue avec un revolver et que si on vient le provoquer, il saura s'en servir"7 , etc.... Seulement la violence qui, de fait, s'exprime à Paris les premier mai 1919 et 1920 ou lors des grèves de Brest et de Vienne en particulier ${ }^{8}$ prend exclusivement pour cible ces agents de l'appareil d'Etat que sont les forces de l'ordre et évite toute autre incarnation, (fût-elle symbolique) plus centrale. La SFIO qui appelait à manifester "contre le verdict et pour honorer Jaurès"g après l'acquittement de Villain avait organisé, en dépit de l'ordre des facteurs énoncés, un cortège identitaire qui ne prenait aucunement pour cible le Palais de justice; les libertaires qui investissent ce cortège ne tentent à aucun moment d'en détourner le cours; la manifestation parisienne du premier mai 1919 n'essaie pas davantage de forcer les barrages protégeant les édifices publics et s'éloigne, au contraire, d'eux, la journée durant, pour élever dans l'Est parisien des barricades qui ne répondent à aucun autre impératif stratégique que l'occupation de la rue. "C'est dommage qu'on n'ait pas eu d'armes car les soldats ne nous auraient pas empêché de passer" déclarent, de leur côté, les dockers de Brest en juin 1919 mais leur objectif ne parait déterminé que par l'interdiction qui leur est faite de l'atteindre et la rue dont ils se réclament ne mène nulle part et du moins pas au Palais d'Hiver. Et seul un dirigeant syndicaliste de Bordeaux pour qui l'action révolutionnaire consiste "à descendre dans la rue avec des revolvers, des fusils, des mitrailleuses en renversant ce gouvernement de bandits"10 parait conférer alors à la rue (et à la violence, tenue pour synonyme) une fonction stratégique. Sous l'espèce, notons le pourtant, d'une simple définition. Ajoutons que la violence que subit alors le mouvement ouvrier ne constitue aucunement le levier escompté pour entraîner les masses dans des voies plus radicales. La riposte à la mort de deux manifestants le premier mai 1919 à Paris puis de trois autres le premier mai suivant en apporte la preuve. Le principe d'une grève générale, envisagée un temps en 1919 est abandonné et les obsèques dont l'Union des syndicats de la Seine est le maître d'oeuvre s'inscrivent pleinement dans la conception socialiste qui ne veut voir en ces morts que ces "grands convertisseurs" invoqués en d'autres temps par George Clémenceau. Ils ne s'accompagnent en mai 1919 d'aucun mouvement de sympathie en province et se déroulent l'année suivante dans une totale discrétion. La violence dont les syndicalistes révolutionnaires se réclament ne prétend donc qu'exceptionnellement à une finalité autre que l'affirmation d'une identité de classe et demeure inscrite à plus d'un titre dans une perspective sorélienne. Elle n'enclenche en aucune manière le cycle provocation/répression/mobilisation escompté par d'aucuns et produit bien au contraire un effet d'autant plus démobilisateur que le "retour à la normale" est partout à l'ordre du jour. La violence perd même dès 1920 toute centralité dans la réflexion de ceux qui l'avaient jusqu'alors revendiquée et disparait simultanément de la scène 
parisienne où son exercice a toujours une portée ou une intention plus stratégique qu'ailleurs $^{11}$. Les manifestations qui rythment traditionnellement les longs mouvements de grèves demeurent par contre, en province, la fréquente occasion d'une violence verbale qui s'exerce à l'encontre du patronat en revêtant d'abord et avant tout une dimension carnavalesque ${ }^{12}$ quand il ne s'agit pas d'une violence parfois moins symbolique qui s'en prend aux jaunes et/ou aux usines refusant de fermer leurs portes. Ces manifestations qui doivent à leur dimension traditionnelle de jouir d'une tolérance plus forte que les démonstrations politiques sont donc l'occasion d'une violence au quotidien dont les manifestants sont cette fois les évidents initiateurs mais dont les effets sont rarement dramatiques ${ }^{13}$. Les organisations syndicales responsables qui le plus souvent sont adhérentes à la CGTU les tiennent, du reste, pour un épiphénomène coutumier dont on ne saurait s'émouvoir ${ }^{14}$. Les maires ou plus souvent les préfets qui ne peuvent tolérer très longtemps ces atteintes à la liberté du travail, interdisent pourtant le plus souvent après quelques semaines ce qu'ils toléraient en début de grève et mobilisent des forces susceptibles de faire respecter les interdits en enclenchant alors presque toujours le processus qui conduit à l'émergence d'affrontements violents avec les forces de l'ordre. C'est ce qui se produit en 1922 lors de la grève des métallurgistes du Havre où les manifestations qui succèdent aux manifestations changent brutalement de caractère après que le maire ait été démis de ses pouvoirs de police et que tous les rassemblements, fût-ce en lieu clos, se soient vus interdire. Des barricades se dressent et de violents affrontements se produisent au cours desquels quatre ouvriers trouvent la mort le 26 août. Le gouvernement et les grands organes de presse soutiennent la thèse d'une agression préalable des militants ouvriers qui aurait mis les forces de l'ordre en situation de légitime défense et les aurait contraint à tirer ${ }^{15}$. Ils prennent argument de ces barricades qui resurgissent quand on les croyait remisées au magasin des accessoires pour dénoncer le péril politique que ferait courir la manifestation en agitant le spectre de la Commune ${ }^{16}$. Les syndicats unitaires qui sont depuis l'origine à la tête du mouvement ne reprennent pas en compte cette comparaison qui, sous leur plume, deviendrait pourtant positive et permettrait d'inscrire la grève dans une tradition de hauts faits. La Vie Ouvrière ne confère à ces barricades incriminées d'autre fonction que défensive ${ }^{17}$. L'Humanité récuse quant à elle la thèse gouvernementale en prenant appui sur la sociologie des victimes pour imputer aux forces de l'ordre la pleine et entière responsabilité des événements ${ }^{18}$ et inscrit la grève du Havre dans la litanie déjà longue de la violence subie ${ }^{19}$. Et c'est en invoquant pareillement Fourmies que Georges Laffont interpelle alors le gouvernement sur ces événements ${ }^{20}$. Les syndicalistes révolutionnaires rendent en septembre 1922 hommage à Sorel récemment disparu en rappelant qu'il "montra la valeur morale de la violence prolétarienne, en analysa l'importance historique, affirma qu'elle pouvait seule préserver le monde de la déchéance intellectuelle et morale à laquelle le conduisait infailliblement les idées et méthodes de lâcheté vers lesquelles les social-réformistes cherchaient à les entraîner"21 mais ils développent dans le même temps une conception défensive de la violence refusant de l'assumer et préférant en accuser l'adversaire. Cette conception cohabite, quelques années durant, avec des appels occasionnels à une violence toujours différée qui joue le rôle catalyseur de tout mythe sorélien ${ }^{22}$ ou avec d'autres qui la réduisent à n'être plus que l'appui, parfois nécessaire, à des démarches à caractère pétitionnaire ${ }^{23}$. 


\section{LES COMMUNISTES}

5 La victoire du fascisme italien et les risques d'une menace fasciste qui pèserait sur la France, amène les communistes à se réclamer de la violence physique à partir de $1923^{24}$. Cette violence revendiquée s'exerce effectivement dès la fin 1924 contre les "cléricalofascistes" qui doivent à la victoire du Cartel des gauches de se mobiliser en privilégiant, quant à eux, la force sur la violence. Elle est, en pratique, tout aussi bien le fait des communistes que des socialistes et autres manifestants anticléricaux et se solde à trois reprises par la mort de militants catholiques ou d'extrême droite ${ }^{25}$. Elle s'exprime également à la veille et surtout après l'exécution de Sacco et Vanzetti ${ }^{26}$, à Paris, le 23 août 1927, et dans certaines villes de province, à l'initiative souvent conjointe de militants communistes et unitaires et des comités Sacco Vanzetti regroupant libertaires et réformistes. Cette action violente qui se déploie ainsi sur le terrain politique de 1924 à 1927 ne relève d'aucune théorisation stratégique; elle apparaît dans des "manifestations à chaud", prend appui sur une puissante indignation préalable et produit des effets qui outrepassent toujours les objectifs initiaux des organisateurs, y compris au plan local. La presse réformiste et les élus socialistes prennent en 1927 leur explicite distance vis à vis d'une telle violence jugée préjudiciable à la cause qu'elle entend promouvoir ${ }^{27}$. Le parti communiste se divise à son propos puis finalement désavoue l'exaltation qu'en a fait Vaillant Couturier après la manifestation parisienne du 23 août 1927. Il lui retire la responsabilité de l'Humanité qui cesse alors d'assumer les exactions produites à cette occasion et admet la thèse de provocateurs mêlés aux manifestants. Ce parti demeure fidèle à cette appréciation jusqu'à son sixième congrès. L'adoption par l'Internationale communiste de la tactique dite "classe contre classe" et l'accession du "groupe" de la jeunesse communiste à la direction du parti pour mieux en assurer la mise en oeuvre renverse la perspective ${ }^{28}$. $L$ 'internationale communiste qui souhaite engager ses sections dans "des formes d'action supérieure" conformes aux exigences de la "troisième période" cherche à radicaliser leur action et appelle, à ces fins, à "conquérir la rue". Elle invite ses sections et la section française en tout premier lieu à développer une: "la violence révolutionnaire [seule] en mesure de briser la violence réactionnaire" ${ }^{29}$ et les engage dans un processus de "manifestation permanente" qui doit culminer avec la "journée rouge" du premier août $1929^{30}$ et dans lequel chaque démonstration est conçue comme propédeutique à la démonstration suivante. Jusqu'à la révolution ou pour en précipiter le cours? Une question qui n'est jamais posée en ces termes dans une presse pourtant prolixe et à laquelle le parti répond en juxtaposant des définitions parfois contradictoires. Le gouvernement qui tient l'énoncé de "la conquête de la rue" et de la violence qui en est le corollaire pour synonyme d'insurrection ${ }^{31}$ prend toutes les dispositions utiles pour les annihiler et réussit au delà de toute espérance. Le PC l'accuse de lui attribuer des objectifs qui n'étaient pas les siens pour mieux pouvoir a posteriori souligner son échec. S'agit-il de passer de cette dénégation à une approche positive des pratiques dont il se réclame, et des définitions émergeant alors de son discours. La première définition nous est suggérée par les références historiques qu'il mobilise. Il récuse le blanquisme, invoque, comme le fait l'Internationale, les premiers mai russes d'avant 1914, le premier mai 1929 à Berlin ou les événements de Vienne et de Canton mais leur adjoint la Commune, les grèves de 1905 à Limoges, les premier mai parisiens de 1919 et $1920^{32}$ et exemplarise, enfin, à diverses reprises l'action des terrassiers ${ }^{33}$. Ce retour en force de la geste 
anarcho- syndicaliste et de ses acteurs majeurs s'accompagne d'une réévaluation de la manifestation parisienne du 23 août 1927 précédemment évoquée. Les cibles que le parti désigne explicitement et les moyens qu'il préconise pour les atteindre confortent la thèse du modèle anarcho-syndicaliste dès lors que les manifestations qui, de nouveau, se réclament de la violence ne posent (dans leurs formes ou leurs objectifs) la question de l'appareil d'Etat qu'en le réduisant à ses seuls agents répressifs. Là s'arrête pourtant la similitude. La rue se voit en effet conféré des objectifs d'abord et avant tout pédagogiques qui font d'elle le terrain privilégié d'une gymnastique révolutionnaire. Le parti qui constitue alors la combativité physique des militants en indice de leur conscience de la classe tient la violence dont ces manifestations sont l'occasion pour un moyen privilégié de séparer le bon grain de l'ivraie ${ }^{34}$. Il y voit encore, et peut être surtout, le possible révélateur d'une violence de classe susceptible de donner corps aux théories du "social-fascisme" et de la troisième période ${ }^{35}$. Il comprend ainsi la violence sous une double espèce et dénonce la violence bourgeoise qui s'exerce à l'encontre de la classe ouvrière tout en exaltant la violence prolétarienne dont celle ci peut, et doit, devenir l'auteur en voyant en elle l'incarnation de la classe en majesté. Il se garde en conséquence d'entraver la répression et souhaite au contraire la provoquer et l'amplifier pour ainsi précipiter la "radicalisation des masses" attendue. Son bureau politique déplore, à ce titre, que les militants de la région parisienne se soit laissés appréhender le premier mai 1929 par des agents qui "avaient reçu des instructions les invitant à être très convenables avec les camarades arrêtés" ${ }^{16}$ et appelle désormais à s'opposer aux arrestations préventives. "Se rendre sans résistance, c'est capituler". Il déplore que certaines manifestations demeurent autorisées ${ }^{37}$, se félicite d'avoir contraint, le premier août, le gouvernement a mettre Paris en état de siège ${ }^{38}$, salue à diverses reprises "la meilleure résistance à la police" et franchit un pas supplémentaire le premier mai 1930 en constituant en région parisienne des "brigades volantes" ayant pour mission d'amener la police à réagir contre elles afin d'amplifier la riposte, "selon une méthode employée avec succès à Berlin" ${ }^{19}$. Le nombre des militants arrêtés après chaque manifestation devient dans une telle perspective un communiqué de victoire . S'agit-il de déterminer les finalités dernières de ces manifestations et les dénégations quant au caractère insurrectionnel de certains modèles $^{40}$ voisinent avec des proclamations plus ambiguës: "La libération prolétarienne est une oeuvre de longue haleine, écrit Gabriel Péri après le premier mai 1929 à Berlin. Les communistes sont résolus à l'accomplir jusqu'au bout. Ils ne l'accompliront qu'en opposant à la violence bourgeoise la violence prolétarienne, en perfectionnant au cours d'escarmouche pareille leur capacité de combat"41. "Le prolétariat chassé de la rue parce qu'il est désarmé y reviendra les armes à la main renchérit un peu plus tard La Journée Rouge. Si auparavant, conquérir la rue signifiait pour le prolétariat européens conquérir les libertés élémentaires, maintenant la reprise de la rue conduit à l'insurrection armée contre l'Etat capitaliste, à la lutte pour le pouvoir prolétarien" ${ }^{42}$. Ces contradictions nous paraissent moins relever d'un quelconque "double langage" ${ }^{43}$ que d'une difficulté à définir ce qui ne peut trouver place dans le système politique français. Nous en voulons pour preuve la très longue définition de la "journée rouge" que donne L'Humanité": "La journée du premier août doit être une étape [...] de la bataille prolétarienne [...] contre l'impérialisme, écrit-elle, une revue de force du prolétariat et de ses alliés face à la guerre qui vient. Elle n'est pas la révolution ni l'insurrection mais en constituera un chaînon dans le sens de l'organisation au sein des usines et dans les villages du plus grand nombre d'ouvriers et de paysans en vue de la lutte permanente 
contre la guerre et le gouvernement qui la prépare. Elle n'est pas l'émeute mais l'organisation consciente de la résistance [...] à la répression [...]. Elle n'est pas le putsch mais la journée où les prolétaires cesseront le travail et manifesteront [...] pour leurs revendications immédiates, leur droit à la rue en même temps qu'ils affirmeront leur volonté puissante de lutte contre la guerre impérialiste et la solidarité avec l'URSS" ${ }^{44}$. Les manifestations que le parti organise dans cette perspective nouvelle sont, de fait, des mouvements sans interlocuteur ni cible véritable. Elles ne relèvent pas bien sûr de la révolte dès lors qu'elles sont le produit d'un volontarisme sans précédent mais ne cherchent pas davantage à marcher sur les lieux d'exercice du pouvoir ou sur des objectifs qui permettraient d'en neutraliser l'action ${ }^{45}$. Elles se veulent "un pas dans la préparation de la transformation de la prochaine guerre impérialiste en guerre civile" 46 et s'inscrivent donc dans une stratégie de défaitisme révolutionnaire (qui suppose la guerre) en cherchant simplement à créer les conditions (psychologiques et politiques) de la révolution ainsi comprise. Le parti communiste que le gouvernement nous l'avons vu tient en échec total dans la capitale exemplarise certaines manifestations violentes qui se déroulent alors même en province. Ainsi en va-t-il des barricades qui s'élèvent à Limoges en juin 1929 lors du procès d'un "bourgeois aux moeurs particulières", de la manifestation au cours de laquelle des manifestants s'en prennent nuitamment à la gendarmerie de Montereau, les manifestations organisées à Troyes et à Lyon à la veille du premier août 1929. Il exemplarise pareillement certaines grèves dont les objectifs explicites n'ont que de lointains rapports avec les siens mais qui du moins donnent lieu à de vifs affrontements avec les forces de l'ordre : ainsi "les dockers qui se sont battus avec les gendarmes", la grève de Concarneau "qui fût une véritable guerre avec les gendarmes" ou les grèves de la Loire qui "témoignent d'une combativité ouvrière de beaucoup supérieure à celle du parti"47. Il exemplarise enfin, à un degré jusqu'alors jamais atteint, les barricades qui se dressent le 13 juin 1931, rue des Longues Haies à Roubaix pendant la longue grève du textile $d u$ Nord $^{48}$. Une brochure intitulée Les barricades de la rue des Longue Haies et préfacée par Maurice Thorez est aussitôt éditée dans l'objectif avoué d'ainsi préparer la journée du premier août 1931. "Les barricades portent la lutte de classe à son niveau le plus élevé, y écrit le secrétaire du parti qui renoue ainsi avec une conception offensive du phénomène barricade, "c'est l'arme de la glorieuse Commune de Paris, de l'insurrection de Moscou en 1905, de la révolution bolchevique, des luttes révolutionnaires d'Allemagne. Les barricades de Roubaix en 1931 sont le prolongement de celles de Berlin en 1919" et de convoquer à l'appui Jules Guesde qui déclarait en 1882 : "Après avoir fait révolution sur révolution, après s'être affranchi politiquement à coups de fusil, la bourgeoisie refuse au prolétariat en travail d'affranchissement le droit aux barricades dont elle a usé et abusé. Ce qui était honneur et devoir pour elle est devenu un crime pour nous" ${ }^{49}$. Cette violence agissante et assumée ne disparaît pas avec le "tournant" communiste d'août 1931 qui vaut pourtant au discours, et à certaines pratiques, du parti de se transformer ${ }^{50}$. Elle resurgit pendant la grève du textile de Vienne de mars-avril 1932 et se voit pleinement assumée par l'Humanité qui inscrit explicitement les barricades dont elle est de nouveau l'occasion dans la filiation de celles de Roubaix. Elle se manifeste encore pendant la grève générale de Strasbourg d'août $1933^{51}$ puis dans des formes suffisamment similaires pour que nous les intégrions alors elles débordent pourtant le cadre chronologique que nous nous étions initialement imparti le premier mai 1934, dans les HBM d'Alfortville et cité Jeanne d'Arc dans le 13eme arrondissement de Paris. Et il faut attendre 1935 pour que resurgisse un discours défensif sur telle ou 
telle barricade qui persiste à s'élever ${ }^{52}$; dans une conjoncture politique nouvelle qui implique que l'on prenne ses distances vis à vis de toute action violente ${ }^{53}$.

\section{VIOLENCE COLERIQUE OU STRATEGIQUE}

7 L'effective responsabilité du parti dans la violence qu'il exemplarise ou du moins assume de 1929 à 1934 fait question. Les manifestations qui précèdent le 1er août 1929 sont assurément conçues comme autant de moyens destinés à préparer le premier août, mais l'émergence d'une violence effective parait assez peu devoir à une quelconque intervention volontariste relevant d'un projet préconçu : les autorités qui tendent alors à voir partout la main du parti reconnaissent le caractère confus et largement spontané des événements de Limoges, la violence naît à Troyes et à Lyon de l'indignation suscitée, parmi les militants communistes, il est vrai, par des arrestations préventives et les exactions dont certaines grèves sont l'occasion effective s'inscrivent dans une longue tradition qui doit peu à la conjoncture. D'où le caractère non reproductible de ce qui se veut pourtant un exemple dès lors qu'on sort du cadre ayant permis ces explosions de "violence colérique" ${ }^{54}$ pour intégrer celui des "journées" décidées à un niveau international. La technicité des barricades de Roubaix et de Vienne, la reproduction de leurs plans par l'Humanités5, le choix du quartier populaire des Longues Haies où s'entassent 10000 personnes parmi lesquelles de nombreux étrangers et qui est "le seul quartier de Roubaix que les allemands ne se soient pas souciés d'occuper durant la guerre" ${ }^{15}$ ainsi que la publication simultanée par les éditions communistes de L'Insurrection armée de Neuberg plaide au contraire en faveur de mouvements sciemment préparés ${ }^{57}$. Les choses sont moins évidentes à Strasbourg en 1933 où la presse communiste assume la violence sans toutefois l'exalter ${ }^{58}$. Les événements du premier mai 1934 qui ne s'inscrivent dans aucune manière dans la conception initiale de la journée ${ }^{59}$ mobilisent du moins, quant à eux, ces même techniques que sont le repli sur des îlots sociologiquement favorables et l'érection de barricades. Les barricades érigées dans ces diverses circonstances se veulent d'abord et avant tout destinées à protéger les manifestants contre les charges des forces de l'ordre. Que la presse communiste les érige en modèle au point d'en reproduire certains plans amène toutefois à s'interroger sur leurs éventuelles finalités stratégiques. Une éphémère revue communiste se donnant pour "l'organe historique et théorique des insurrections" rendait en 1927 hommage à Blanqui en soulignant sa "compréhension du rôle militaire de la violence" et reproduisait, croquis à l'appui, ses écrits relatifs aux barricades ${ }^{60}$. Les barricades qu'on vient d'évoquer ne sauraient en aucune manière se prévaloir d'un tel rôle. "Ici c'est la frontière du peuple : les assassins ne passent pas" proclame une pancarte fichée sur la barricade de la rue des Longues Haies, largement reproduite par la presse communiste. La proclamation nous parait parfaitement significative de la stratégie de repli sur des fortins que l'Humanité appelle à multiplier ${ }^{61}$; pour marquer des positions dans l'attente d'une crise révolutionnaire qui suppose, on l'a vu, d'autres détonateurs. Que le parti communiste multiplie de 1929 à 1931 les références à la geste syndicaliste révolutionnaire ne doit dès lors pas faire illusion. La violence dont il se réclame s'inscrit dans la perspective du défaitisme révolutionnaire et non plus dans celle de la grève générale. Que ces stratégies profondément distinctes ne soient pas plus insurrectionnelles l'une que l'autre répond 
toutefois de certaines similitudes quant aux cibles de la violence et à leur imprécision ; elle explique les ressemblances entre ces barricades dressées dans des rues qui ne mènent jamais nulle part et qui s'inscrivent, au demeurant, dans des villes jouissant en la matière d'une tradition depuis l'avant guerre ${ }^{62}$. Elle répond également de la faible validité du modèle blanquiste ${ }^{63}$. Le discours tenu sur la violence prolétarienne nous parait en dernière analyse remplir le rôle de mythe substitutif à celui de la grève générale; au prix d'un déplacement de l'usine vers la rue et malgré le caractère antagonique de ces stratégies censées les sous-tendre. Par quoi le rapport communiste à la violence relèverait alors moins du syndicalisme-révolutionnaire en acte que des conceptions soréliennes nonobstant la stratégie dont il se réclame explicitement. La violence effective s'inscrit quant à elle d'abord et avant tout dans une tradition de lutte ouvrière qui anticipe toute théorisation. Elle demeure, ces années durant, globalement conforme aux réflexions soréliennes qu'elle a suscitées et conserve une assise géographique, et nous semble-t-il sociologique ${ }^{64}$, héritée de l'avant guerre. Le parti communiste qui tente de mettre en oeuvre une stratégie d'action violente s'inscrivant dans une théorie dont l'extériorité vis à vis du système politique et social prévalant en France est importante se voit réduit à exemplariser des mouvements qui nous paraissent relever largement de cette première conception ; quand bien même il en est, parfois, l'initiateur. Cette violence dont la dimension culturelle nous semble, en conclusion, beaucoup plus forte que la dimension stratégique ne disparaît du reste pas avec la stratégie censée l'avoir suscitée. Elle demeure toutefois, de 1919 à 1934 et à l'échelle nationale, un phénomène plus spectaculaire qu'efficient. Ajoutons que cette violence théoriquement revendiquée qui est d'autant plus spectaculaire qu'on lui assigne des fins exemplaires est la mieux maîtrisée par les forces en présence. Elle est, dès lors, moins coûteuse en vie humaine ${ }^{65}$ que cette autre violence, plus spontanée, qui met aux prises manifestants et contre-manifestants; sans théorisation préalable ni objectifs stratégiques, de 1924 à 1926 comme après 1934.

\section{NOTES}

1. G. Sorel, Réflexions sur la violence, Marcel Rivière, 1921, p.257 et 263.

2. AN F7 13273, rapport du 22 avril 1919 : Péricat envisage ainsi que la grève du premier mai puisse se poursuivre en entraînant "des bagarres, des émeutes et la Révolution".

3. APP, Ba 1628, réunion rue de Cambronne, 30 avril 1919.

4. AN F7 13273, meeting des cheminots à la bourse du travail, 30 avril 1919.

5. AN F7 12992, réunion du syndicat des métaux de Grenoble, 9 décembre 1918.

6. Une manifestation de solidarité avec la Russie prévue ce jour sera finalement annulée.

7. AN F7 13015, CE de l'Union des syndicats de la Seine, 16 juillet 1919.

8. Juin et septembre 1919.

9. L'Humanité, 5 avril 1919.

10. AN F7 13367, syndicat des mécaniciens de Bordeaux, 2 novembre 1919. 
11. Danielle Tartakowsky, "La politique et la rue", Ethnologie Française, 1991, N³, pp. 317-322. Deux manifestants trouvent toutefois la mort au sortir d'un meeting le 8 mai 1921 et lors de la dispersion d'un autre meeting le premier mai 1923 à Paris. Un mort également dans des circonstances identiques à Suresnes lors de la grève générale du 12 octobre 1925 contre la guerre du Maroc.

12. Dans le textile du Nord en particulier.

13. Deux exceptions : un gréviste meurt à Elboeuf, en avril 1923, d'un arrêt cardiaque après une bagarre avec des jaunes ; même phénomène lors de la grève des chauffeurs de taxis en janvier 1934.

14. La Vie Ouvrière, 1er septembre 1922, à propos de la grève du Havre : "L'ordre n'était nullement troublé. Quelques incidents, habituels en période de grève (souligné par nous), s'étaient produits entre ouvriers grévistes et quelques inconscients qui avaient refusé de se joindre au mouvement".

15. Les photos que publie l'Excelsior des 27 et 28 août 22 s'emploient à fonder cette thèse : on y voit des "chômeurs" dépavant le cours de la République puis lançant des pavés sur les forces de l'ordre.

16. AN F7 13934, rapport préfectoral du 31 août 22 : "Si le commissaire central du Havre avait faibli samedi soir, la "Commune" s'installait au Havre d'où il aurait fallu la déloger. A quel prix ?".

17. La Vie Ouvrière, premier septembre 1922 : "Les grévistes prennent des mesures nécessaires pour se défendre (souligné dans le texte) et font en sorte que de nouvelles charges ne se produisent pas".

18. L'Humanité, 27 août 1922 : "L'agence Havas parle de 800 coups de revolvers tirés sur la police mais c'est du coté ouvrier qu'on compte 3 morts". Un quatrième militant décède ultérieurement de ses blessures.

19. La déclaration de la CGTU rappelle "Fourmies, Raon l'étape, Draveil, Villeneuve Saint Georges" et celle de la CGT "Limoges, Chalon, Narbonne, Draveil, Villeneuve Saint Georges".

20. Fourmies est pareillement invoqué par La Vie Ouvrière lors de la grève du textile de l'Isère d'août 1922 après une charge qui cette fois n'est pas meurtrière (18 août 1922). Renoult l'invoque pareillement quand il interpelle le gouvernement sur les incidents ayant marqué la grève des sardinières de Douarnenez en 1925.

21. G. Louzon, La Vie Ouvrière, 2 septembre 1922.

22. AN F7 13587, Bourges, rapport sur la "journée" du 7 novembre 1926 : "Vous nous avez eu cette fois mais nous aurons des matraques et des revolvers la prochaine fois" ; Dijon : "Les manifestations ne seront pas toujours pacifiques, les ouvriers descendront dans la rue avec des fusils", Ibid,

23. AN F7 13643, Var, rapport du 9 juin 1922 sur une réunion du conseil syndical des marins : "Lorsque nous aurons conspué le préfet maritime et que nous nous serons bousculé avec quelques flics, peut-être les pouvoirs publics daigneront-ils s'émouvoir de notre situation".

24. L'Humanité 22 juin 1923 : les travailleurs doivent "absolument en imposer à une force oppressive dont la hardiesse ira croissant si dès le début de ses exploits, la virilité (sic. DT) de la classe ouvrière ne la rappelle énergiquement à la prudence élémentaire". 25. Marseille, manifestation de la FNC, 1925 ; attentat de la rue Danrémont, 1925, Saint Etienne 1926.

26. Des barricades s'élèvent à Paris où des pillages sont en outre $\mathrm{p}$ perpétrés, des gendarmes sont blessés à Saint Nazaire et Dunkerque et agressés par des militants 
dotés d'armes à feu à Biarritz. Des manifestants sont blessés à Lyon et au Havre, des coups de feu tirés à Besançon et des barricades s'élèvent à Cherbourg.

27. Paul Faure rappelle alors que les communards ont traîné pendant 50 ans l'accusation de pétroleurs.

28. Stéphane Audoin, "le parti communiste et la violence", Revue historique , $\mathrm{N}^{\circ} 546$, avril-juin 1983.

29. L'Humanité, 7 avril 1929, intervention de "Williams", délégué de l'internationale au congrès de Saint Denis.

30. Puis des premiers août 1930 et 1931.

31. APP Ba 1716, rapport sur l'action publique et clandestine du PC en vue de faire du premier août une journée insurrectionnelle : "Cette étude est établie sur des documents qui ont été livrés à une si large publicité qu'on serait tenté d'en nier l'importance. [...] Il n'est pas douteux qui si ces textes avaient été découverts au cours de perquisition, sous forme de correspondances particulières ou collectives et marquées d'un sceau confidentiel l'existence d'un complot n'aurait fait de doute pour personne".

32. AN F7 13296, tract de l'USRP pour le premier mai 1931 : "Vous redonnerez au premier mai l'allure de bataille de masse des premiers mai d'avant guerre et de 1919-1920".

33. IML, bobine 58 , compte rendu du bureau politique Sémard, après le premier mai 1929 : "Il y a eu le premier mai des redditions volontaires en masse alors qu'autrefois 50 gars du bâtiment devant 10 flics les auraient fichu en l'air". Directives pour la manifestation du 25 février 1930 : "Pour encourager les camarades, annoncer que les syndicats du bâtiment seront représentés par 500 gars décidés".

34. AN F7 13296, les carnets du militant $\mathrm{N}^{\circ} 3$, "l'autodéfense pour le premier août" : "l'activité d'ici cette journée et pendant la journée sera une base pour juger de la qualité et de la force révolutionnaire des membres du parti".

35. D'où l'importance qu'il accorde au premier mai 1929 à Berlin où les ouvriers se sont heurtés à une répression meurtrière à l'instigation du préfet socialiste. L'Humanité du 21 juin 1931 se félicite dans cette même perspective qu'une "très grande haine [ait] surgi [à Roubaix] contre la police et les mobiles".

36. IML, bobine 58, compte rendu du bureau politique, Sémard : "Cela se passait en famille. les camarades ont pris les arrestations pour une rigolade avec les flics".

37. AN F7 13322, rapport du 28 mai 1929 sur le Mur des fédérés : "Les dirigeants de la jeunesse communiste pensent qu'il "eût été préférable que la manifestation soit interdite. Cela aurait permis d'organiser des colonnes qui auraient provoqué des bagarres dans différents points de Paris".

38. L'Humanité,14 août 1929, déclaration du comité central sur le premier août 1929 : "La classe ouvrière a pu se rendre compte des méthodes qu'emploiera la bourgeoisie au moment de la déclaration de guerre, elle a dû abattre son jeu. On ne saurait parler d'un échec du PC dès lors que la bourgeoisie est réduite à employer de tels moyens pour empêcher la moindre manifestation".

39. IML, bobine 53, réunion du BP du 26 mars 1930.

40. AN F7 13296, bureau occidental de l'IC, Berlin 16 mai 1929 : "Partant du point de vue que nous ne sommes pas dans une situation révolutionnaire ; [le parti allemand] n'a pu se laisser entraîner dans le piège comme en 1921". [la ligne du parti était] : "lutte contre l'interdiction des manifestations du premier mai, lutte pour la conquête de la rue, démonstrations de rue non armées, pas de lutte armée mais résistance à la police. Ces mots d'ordre furent suivis. Ce n'est que dans la soirée que commencèrent à s'édifier 
des barricades dans les quartiers ouvriers pour empêcher les flics du social-fasciste Zorgiebel de pénétrer dans leur quartier".

41. L'Humanité, 3 mai 1929.

42. AN F7 13301, rapport général sur le premier août 1929.

43. Nous divergeons ici des conclusions de Stéphane Audoin, op. cit. p.371.

44. L'Humanité, 12 juin 1929," le caractère et la signification du premier août".

45. Il est toutefois parfois question de neutraliser que le service d'ordre : Ba 1716, note du 18 juillet 1929, rapport sur l'action publique et clandestine du PC en vue de faire du premier août une journée insurrectionnelle : "Que le service d'ordre soit un jour défaillant et c'est la conquête de la rue assurée, c'est la révolution maîtrisée, c'est la dictature du prolétariat".

46. Ba 1716, note du 18 juillet 1929 : rapport sur l'action publique et clandestine du PC en vue de faire du premier août une journée insurrectionnelle : intervention de Sémard au CC clandestin d'Achères.

47. IML, bobine 47, comité central du 20 juin 1929, rapport de Célor.

48. Signalons que ces barricades certes remarquables par leur technicité ne sont pas chose exceptionnelle. Elles surgissent à Limoges lors de l'épisode déjà cité, à Rouen le 27 juillet 1930 lors d'une manifestation des syndicats unitaires contre les assurances sociales et à Roubaix une première fois le 29 août 30 .

49. Maurice Thorez, Oeuvres, Editions sociales, 1950, livre deuxième, tome 1, pp 225-227.

50. Nous divergeons ici une nouvelle fois de de l'article de Stéphane Audoin déja cité. 51. Les communistes et les unitaires récusent alors la thèse d'une action provocatrice de groupes autonomistes d'obédience nazie mise en avant par les autorités.

52. Grève des gantiers de l'Aveyron, janvier 1935 ; grève de Trith saint Léger, 29 janvier 1935, grève de Toulon, août 1935.

53. Danielle Tartakowsky, "Stratégies de la rue, 1934-1936", Le Mouvement social, $\mathrm{n}^{\circ} 135$, avril-juin 1986, pp.31-62.

54. Nous empruntons ce terme débattu lors de la table ronde des 24 et 25 septembre au rapport introductif de Philippe Braud.

55. Elle reproduit une nouvelle fois le 2 mai 1934 le schéma de la barricade d'Alfortville. 56. AN F7 13920, rapport du 15 juin 1931.

57. AN F7 13920, rapport du 29 juin 1931, information parvenue de Genève : "La main de Moscou était nettement visible ; les personnes qui ont organisé les désordres dans la rue connaissaient certainement la tactique de combat. Avant tout c'est le choix du lieu des désordres qui confirme la supposition. La presse communiste qui n'est disconvient pas totalement fait du moins des "prolétaires" et non de l'organisation le sujet de l'histoire".

58. AN F7 13935, rapport préfectoral du 4 septembre 1933. Le rapport parle d'une "véritable émeute plus que d'une manifestation qui paraissait organisée supérieurement par des spécialistes de ce genre d'opération selon un plan bien combiné". Il évoque l'action de spécialistes venus de la région parisienne et s'exprimant en français.

59. La CGTU a prévu un rassemblement central dans la clairière de Reuilly. Des incidents se produisent après la dispersion et à la suite de l'interpellation de militants. 60. Le Militant rouge, $n^{\circ} 12,1926$ et $n^{\circ} 1$ et 2, 1927 : "matériaux pour l'étude du blanquisme" 
61. L'Humanité, 21 juin 31 : "Nous avons à notre portée des forteresses mais nous n'avons jamais su les organiser : est ce que nous n'avons pas des longues Haies dans toutes nos localités ?".

62. Paris, Limoges "la rouge", Brest "la rouge" Vienne ( 1882), et, dans le Nord, Armentières (1903) dont la tradition est invoquée à Roubaix.

63. Il convient du reste de préciser que les "matériaux pour l'étude du blanquisme" précédemment évoqués sont des articles du communiste allemand Paul Frölich.

64. Le rôle des ports est en particulier évident.

65. Les manifestations syndicales ayant provoqué mort d'hommes se situent aux deux extrémités de la chaîne chronologique qui est ici la nôtre ; dans des phases où la stabilisation n'est pas totale ou, tout au contraire, remise en question. Quelques décès accidentels se produisent toutefois au cours de grèves mais hors de toute manifestation : ainsi à Port de bouc en 1932, lors de la grève du bâtiment cette même année et pendant la grève des chauffeurs déjà évoquée.

\section{RÉSUMÉS}

Certaines composantes du mouvement ouvrier français se réclament explicitement de la violence à deux reprises, de 1919 au début des années trente. Il s'agit des syndicalistes révolutionnaires au sortir de la guerre puis du Parti communiste entre 1929 et 1931. Ce texte analyse les finalités que ces organisations assignent à la violence, en compare les termes et s'interroge sur les relations qu'entretiennent leur théorisation et la violence effective de certaines manifestations ouvrières qui se déploient ces années durant. Il en ressort que le discours tenu sur la violence prolétarienne remplit, en dernière analyse, le rôle de mythe substitutif à celui de la grève générale ; au prix d'un déplacement de l'usine vers la rue et malgré le caractère antagonique des stratégies censées sous-tendre l'une et l'autre. Par quoi le rapport communiste à la violence relèverait alors moins du syndicalisme-révolutionaire en acte que des conceptions soréliennes, nonobstant la stratégie dont il se réclame explicitement. La violence effective s'inscrit quant à elle, d'abord et avant tout, dans une tradition de lutte ouvrière qui anticipe toute théorisation. Elle demeure, ces années durant, globalement conforme aux réflexions soréliennes qu'elle a suscitées et conserve une assise géographique (et, nous semble-t-il, sociologique) héritée de l'avant guerre.

\section{INDEX}

Mots-clés : émeutes, histoire, mouvements politiques, Mouvements sociaux, villes/ sociologie urbaine

Index chronologique : 20ème siècle 